\title{
Dependence of the Interfacial Behavior of $\beta$-Casein on Phosphoserine Residues
}

\section{M. Cassiano and J. A. G. Arêas}

Departamento de Nutrição, Faculdade de Saúde Pública, Universidade de São Paulo Av. Dr. Arnaldo, 715, 01246-904, São Paulo, Brazil

\section{ABSTRACT}

The role of the phosphoserine residues on the dynamical and structural properties of $\beta$-casein was studied by molecular dynamics of the protein in water/lipid interfacial regions. The initial protein structure adopted in the modeling was that proposed for bovine $\beta$-casein $\mathrm{A}^{2}$, where the five phosphoserine residues, originally present in its primary structure, were partially or totally substituted by serine residues. The simulations revealed a dependence of the interfacial behavior of $\beta$ casein on the phosphorylation grade. When only partially dephosphorylated, the protein showed a similar behavior as that observed for the original $\beta$-casein reported in previous work. During dynamics, the protein migrated from the aqueous environment towards the lipid medium, and remained attached to the interface separating both media. Quite different was the dynamics of the totally dephosphorylated $\beta$-casein, that did not perceive the interface and immersed incessantly into lipid medium. The results suggest that the phosphoserine residues appear to be, in fact, intrinsically related to the mechanisms of $\beta$-casein emulsion stabilization.

(Key words: dephosphorylated $\beta$-casein, molecular dynamics, lipid-protein interaction, functional properties)

Abbreviation key: SerP = phosphoserine.

\section{INTRODUCTION}

Casein is the major group of milk proteins and it is widely used in the food industry as a highly functional ingredient (Hill, 1996). Although it is used mainly for its ability to form and stabilize emulsions, the mechanisms related to the stability and interfacial properties of casein are not completely understood (Husband et al., 1997). The primary structure of the known genetic vari-

Received July 10, 2003.

Accepted September 8, 2003.

${ }^{1}$ Corresponding Author: M. M. Cassiano; e-mail: cassiano@if. usp.br. ants of caseins is well established, and the unique features of their composition are the presence and numbers of phosphoserine (SerP) residues and the high frequency of proline residues (Eigel et al., 1984; Swaisgood, 1992, 1993). Bovine $\beta$-CN A ${ }^{2}$ is constituted by 209 amino acids residues, with charged residues concentrated in the $\mathrm{N}$-terminal region, while the rest of the molecule is mainly hydrophobic, conferring to the protein an amphiphilic nature (Ribadeau Dumas et al., 1972; Eigel et al., 1984; Kumosinski et al., 1993). The frequent statement that $\beta$-CN lacks secondary structure is not correct, since spectral techniques and prediction algorithms indicate that several structural motifs may be present (Swaisgood, 1992, 1993). A new view of protein folding, recently proposed and applied to casein, suggests that the protein has both rigid structures centered on Pro residues and highly flexible elements, represented by loops and helices (Qi et al., 2001; Farrell et al., 2002). Molecular dynamics of $\beta$-CN also indicated the presence of well defined secondary structure in aqueous and lipid media (Arêas et al., 2001; Cassiano and Arêas, 2001). The modeling demonstrated that structuralization is not incompatible with inherent properties of $\beta$-CN. The spatial separation between charged and hydrophobic domains was kept during the dynamics simulations and the amphiphilic behavior of $\beta$-CN was reproduced, observed by the displacement of the protein from the aqueous environment towards the lipid medium and its permanence at the interface separating both media. It was also observed that, after the entrance of the protein in the lipid medium, all SerP residues except SerP35, remained closer than $8 \AA$ from the interface. It has been reported that removal of the phosphate groups from whole $\mathrm{CN}$ reduces its ability to maintain the integrity of emulsions (van Hekken and Strange, 1993). An experimental study on the functional and interfacial properties of $\beta$-CN and dephosphorylated $\beta$-CN suggested that its mechanisms of emulsion stabilization depend on the charged phosphate groups (Husband et al., 1997). The SerP residues, clustered in the N-terminal region of the protein, account for the high net negative charge on this region 


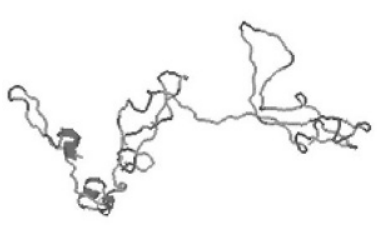

0 ps

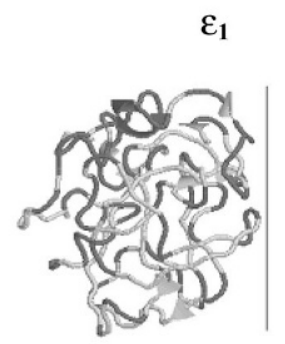

400 ps

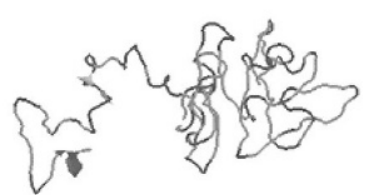

10 ps

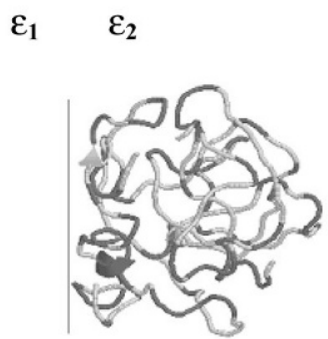

450 ps

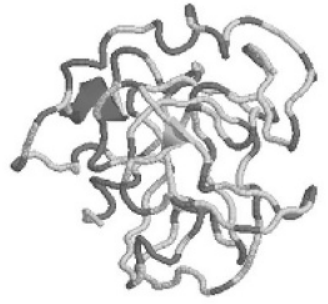

30 ps

$\varepsilon_{1}$

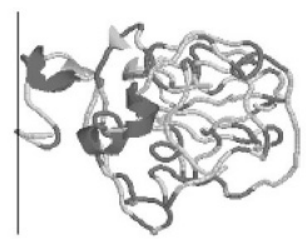

600 ps

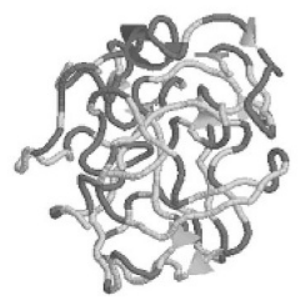

400 ps

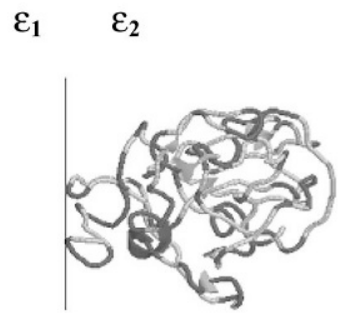

1000 ps

Figure 1. Conformational dynamics of partially dephosphorylated $\beta$-casein in aqueous medium and in the presence of the interface separating aqueous and lipid media, characterized by the dielectric constants $\varepsilon_{1}=80$ and $\varepsilon_{2}=2$, respectively. The views of the molecule are not in the same scale.

and appear to be critical for its emulsion stabilizing properties (Caessens et al., 1999).

In order to verify the dependence of the interfacial and structural behavior of $\beta-\mathrm{CN}$ on the SerP residues and its relationship to the mechanisms of emulsion stabilization, molecular dynamics of partially and totally dephosphorylated $\beta$-CN were performed following the same procedures previously adopted in the simulations of $\beta$-CN reported previously (Arêas et al., 2001; Cassiano and Arêas, 2001).

\section{MATERIALS AND METHODS}

The starting model for the dephosphorylated $\beta$-CN was built with the program SYBYL (Tripos, Inc.), according to the secondary structure proposed in the literature for bovine $\beta$-CN A ${ }^{2}$ (Kumosinski et al., 1993). Two protein models were studied: partially and totally dephosphorylated. In the first model, only SerP residue at position 35 was substituted by serine and in the second model all SerP residues, originally present, were substituted by serine (Ser15, Ser17, Ser18, Ser19, and Ser35). The simulations of molecular mechanics and dynamics were done with program THOR (Mundim et al., 1998), based on GROMOS force field (van Gunsteren and Berendsen, 1987), that permits modeling of proteins in solvents and in water/lipid interfacial regions. Aqueous and lipid media were modeled implicitly as continua, characterized by their dielectric constant, respectively $\varepsilon=80$ and $\varepsilon=2$, and the water/lipid interface was represented by a plane surface separating both media and defining a dielectric discontinuity region (Pascutti et al., 1999; Arêas at al., 2001; Cassiano and Arêas, 2001). The electrostatic interaction between the atoms was, in this case, calculated by the method of electrostatic images (Jackson, 1975; Reitz, 1980). Optimization of initial conformation was done with steepest descent and conjugate gradient algorithms, consecutively (Press et al., 1992). The dynamics was initialized with a gradual heating of the system up to $300 \mathrm{~K}$ in $10 \mathrm{ps}$. This temperature was maintained by a direct rescaling of velocities up to $200 \mathrm{ps}$, and the system was then weakly coupled to an external heat bath, with relaxation time of $0.1 \mathrm{ps}$ (Berendsen at al., 1984). The leapfrog algorithm (van Gunsteren and Berendsen, 1990) was used to perform the integration of the atomic trajectories with a time step of $0.5 \mathrm{fs}$. The simulations were initialized with the protein in aqueous medium and the center of mass coincident with the center of coordinate system. The dynamics showed that equilib- 


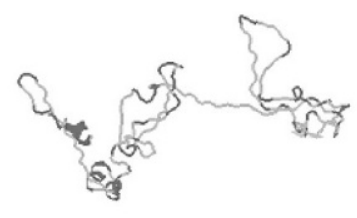

0 ps

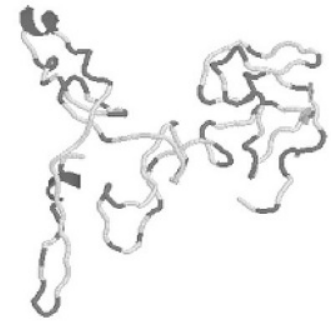

10 ps

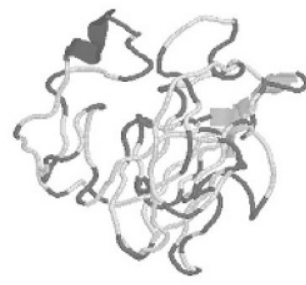

30 ps

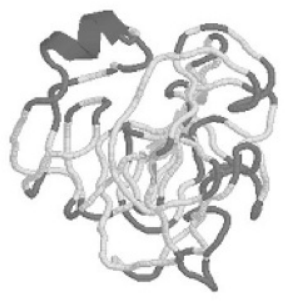

400 ps

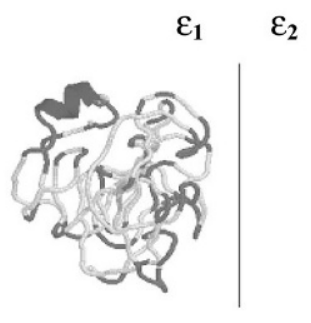

$400 \mathrm{ps}$

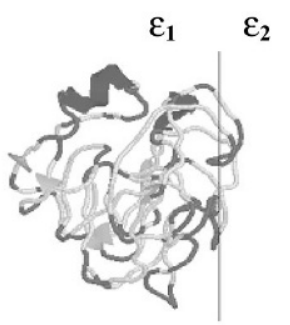

450 ps

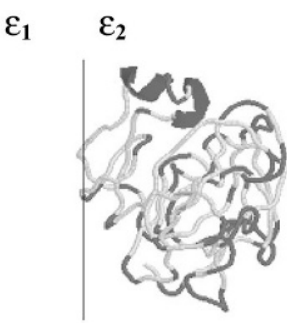

$470 \mathrm{ps}$ $\varepsilon_{1} \quad \varepsilon_{2}$

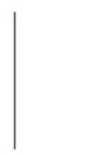

500 ps

Figure 2. Conformational dynamics of totally dephosphorylated $\beta$-casein in aqueous medium and in the presence of the interface separating aqueous and lipid media, characterized by the dielectric constants $\varepsilon_{1}=80$ and $\varepsilon_{2}=2$, respectively. The views of the molecule are not in the same scale.

rium is reached after $100 \mathrm{ps}$. Thus, $400 \mathrm{ps}$ was considered a sufficiently long time interval for the introduction of a plane surface, positioned at about $20 \AA$, for modeling the interface of separation of aqueous and lipid media. Simulations proceeded until a total time of $1 \mathrm{~ns}$.

\section{RESULTS AND DISCUSSION}

Figures 1 and 2 present some conformations, displayed with program RASMOL (Sayle and MilnerWhite, 1995), assumed by partially and totally dephosphorylated $\beta$-CN during dynamics in aqueous medium ( 0 to $400 \mathrm{ps}$ ) and in the presence of a water/lipid interface (400 to $1000 \mathrm{ps}$ ). These figures show that, as observed in previous simulations of $\beta$-CN (Arêas et al., 2001; Cassiano and Arêas, 2001), both partially and totally dephosphorylated $\beta$-CN rapidly evolved from the proposed distended conformation to a more compact one, that was maintained during all the dynamics in aqueous medium. However, the behavior of the protein in the presence of the interface was different in both studied models. Figure 1 shows that when the interface was introduced to the system, partially dephosphorylated $\beta$-CN dislocated to the lipid medium, as already observed for $\beta$-CN (Arêas et al., 2001; Cassiano and
Arêas, 2001). After about 50 ps the protein was completely inserted in the lipid region, attached to the interface, where it remained until the end of dynamics. Unlike native and partially dephosphorylated $\beta$-CN, Figure 2 shows that totally dephosphorylated $\beta$-CN immersed into the lipid medium but did not remain close to the interface, penetrating incessantly into it, indicating its high affinity to this medium. This observation is consistent with the findings of Sood and Slattery (2000) that reported dephosphorylated $\beta$-CN aggregation due to the high hydrophobicity of the molecule.

The trajectory of the system center of mass was followed during dynamics, after the introduction of the water/lipid interface. The behavior of partially dephosphorylated $\beta$-CN (Figure 3) was similar to that observed for the native one. The distance of the protein center of mass to the interface remained around $22 \AA$. Figure 3 also shows the dislocation of the totally dephosphorylated $\beta$-CN in direction to the interface separating aqueous and lipid media and its continuous penetration into the lipid medium.

Structural analysis of partially and totally dephosphorylated $\beta$-CN during molecular dynamics was done with program RASMOL (Sayle and Milner-White, 1995), that uses the DSSP algorithm (Define Secondary Structure of Proteins) (Kabsh and Sander, 1983). Table 


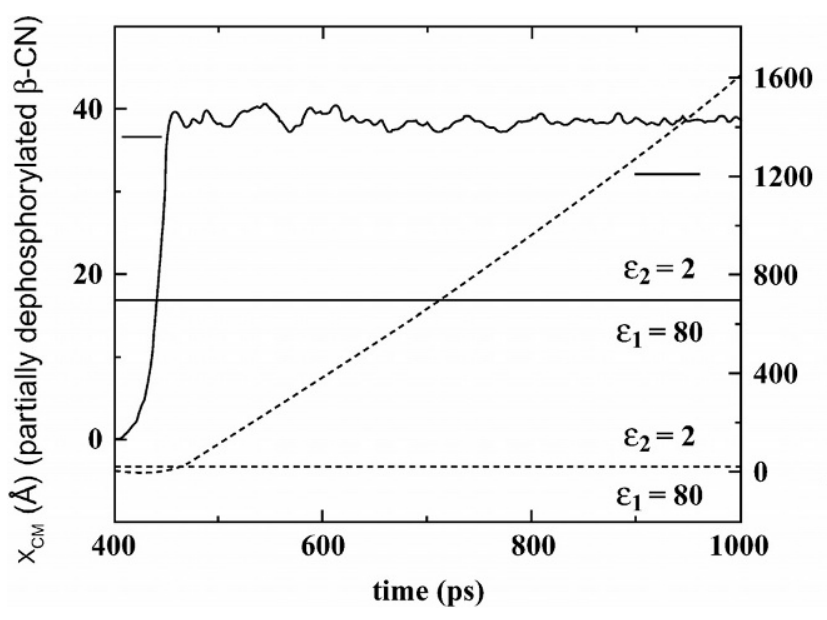

Figure 3. Trajectory of the center of mass (CM) of partially (solid line) and totally (dashed line) dephosphorylated $\beta$-CN in the presence of the water/lipid interface.

1 presents the percentage of amino acid residues in $\alpha$ helices, $\beta$-structures and turns for both protein models, obtained from conformations recorded every $10 \mathrm{ps}$ during the time intervals 200 to $400 \mathrm{ps}$ (aqueous medium) and 800 to 1000 ps (lipid medium). Data of $\beta$-CN from previous work (Arêas et al., 2001; Cassiano and Arêas, 2001) are also presented in Table 1 for comparison. For the 3 models, it was observed a trend of increasing percentage of residues forming $\alpha$-helices and $\beta$-structures when the protein entered into the lipid medium. This is in accordance with the experimental results reporting that lipid and lipid homologues induced an increase in $\alpha$-helix content (de Jongh and de Kruijff, 1990; Rankin et al., 1998). Results obtained by Raman spectroscopy suggest that in water medium $\beta$-CN has about $10 \%$ helical structures, $20 \% \beta$-structures, and 20 to $35 \%$ turns (Byler et al., 1988). Data obtained by circular dicroism and optical rotatory dispersion indicate a proportion of 1 to $20 \% \alpha$-helices and 0 to $13 \% \beta$ structures (Creamer et al., 1981).

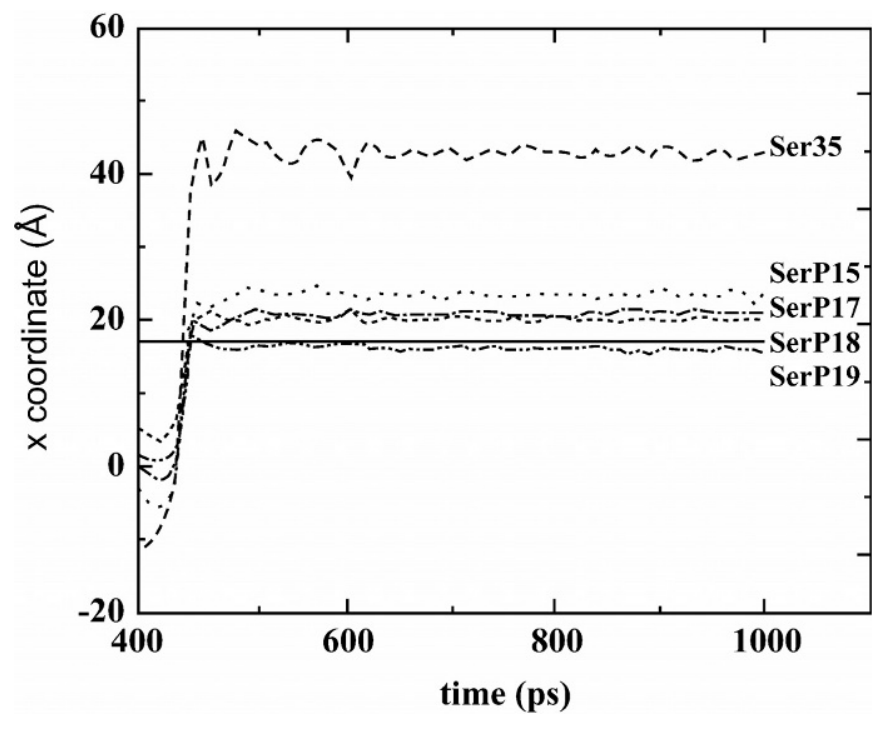

Figure 4. Phosphoserine (SerP) and serine residues positions in relation to the water/lipid interface during dynamics of partially dephosphorylated $\beta$-casein $(\beta$-CN).

The positions of SerP and Ser35 residues were monitored during dynamics of partially dephosphorylated $\beta$ CN. Figure 4 shows that, after the entrance of the protein into the lipid medium, the 4 SerP residues remained closer than $6 \AA$ from the interface. Ser35, that when phosphorylated (SerP35) in the simulation of $\beta$ CN (Cassiano and Arêas, 2001) had already been about $15 \AA$ distant from the interface, stayed even more distant in this simulation, around $25 \AA$. These results reinforce the importance of the SerP residues for maintaining the protein close to the interface and imparting the emulsifying characteristics to $\beta$-CN.

The area occupied by partially dephosphorylated $\beta$ $\mathrm{CN}$ at the water/lipid interface was estimated by considering the projection of all atoms on the plane surface, during the time interval of 800 to $1000 \mathrm{ps}$ of the dynamics. The value of about $315 \mathrm{~m}^{2} / \mathrm{g}$ of protein is comparable

Table 1. Percentage of structures in the conformations of partially and totally dephosphorylated $\beta$-CN during dynamics in aqueous and lipid media. Data of $\beta$-CN from previous work (Arêas et al., 2001; Cassiano and Arêas, 2001) are also included for comparison.

\begin{tabular}{|c|c|c|c|c|c|c|}
\hline & \multicolumn{2}{|c|}{$\beta-\mathrm{CN}$} & \multicolumn{2}{|c|}{$\begin{array}{c}\text { Partially } \\
\text { dephosphorylated } \\
\beta-\mathrm{CN}^{1}\end{array}$} & \multicolumn{2}{|c|}{$\begin{array}{c}\text { Totally } \\
\text { dephosphorylated } \\
\beta-\mathrm{CN}^{2}\end{array}$} \\
\hline & Aqueous & Lipid & Aqueous & Lipid & Aqueous & Lipid \\
\hline$\alpha$-helix (\%) & 4 & 8 & 5 & 6 & 2 & 6 \\
\hline$\beta$-structure (\%) & 7 & 8 & 7 & 13 & $\overline{7}$ & 10 \\
\hline Turns (\%) & 47 & 44 & 45 & 46 & 40 & 39 \\
\hline Total (\%) & 58 & 60 & 57 & 65 & 49 & 55 \\
\hline
\end{tabular}

${ }^{1}$ Phosphoserine residue at position 35 was substituted by serine.

${ }^{2} \mathrm{All}$ phosphoserine residues were substituted by serine. 
to the value $347 \mathrm{~m}^{2} / \mathrm{g}$, obtained for $\beta-\mathrm{CN}$ (Arêas et al., 2001; Cassiano and Arêas, 2001), and it is consistent with experimental values reported in literature for $\beta$ $\mathrm{CN}$ (300 to $400 \mathrm{~m}^{2} / \mathrm{g}$ ), characteristic of good emulsifiers (Graham and Philips, 1979; Hill, 1996).

\section{CONCLUSIONS}

Partially and totally dephosphorylated $\beta$-CN were studied by molecular dynamics, using techniques that permitted modeling of the protein at water/lipid interfacial regions. The results evidenced direct dependence of the interfacial and structural behavior of $\beta-\mathrm{CN}$ on the SerP residues and corroborated the importance of these residues for the maintenance of $\beta$-CN close to the interface. It appears that, in fact, the SerP residues play a fundamental role and are intrinsically related to the mechanisms of emulsion stabilization by $\beta-\mathrm{CN}$.

\section{ACKNOWLEDGMENTS}

The authors thank FAPESP (grants 97/13610-7 and 98/08095-9) for financial support.

\section{REFERENCES}

Arêas, J. A. G., M. M. Cassiano, C. Glaubitz, G. Gröbner, and A. Watts. 2001. Interaction of $\beta$-Casein at an Emulsion Interface Studied by ${ }^{2} \mathrm{H}$ NMR and Molecular Modeling. Pages 193-201 in Magnetic Resonance in Food Science: A view to the Future. G. A. Webb, P. S. Belton, A. M. Gil, and I. Delgadillo, ed. The Royal Society of Chemistry, Cambridge, U.K.

Berendsen, H. J. C., J. P. M. Postma, W. F. van Gunsteren, A. DiNola, and J. R. Haak. 1984. Molecular dynamics with coupling to an external bath. J. Chem. Phys. 81:3684-3690.

Byler, D. M., H. M. Farrell, Jr., and H. Susi. 1988. Raman spectroscopic study of casein structure. J. Dairy Sci. 71:2622-2629.

Caessens, P. W. J. R., H. Gruppen, C. J. Slangen, S. Visser, and A. G. J. Vorangen. 1999. Functionality of $\beta$-casein peptides: importance of amphipathicity for emulsion-stabilizing properties. J. Agric. Food Chem. 47:1856-1862.

Cassiano, M. M., and J. A. G. Arêas. 2001. Study of bovine $\beta$-casein at water/lipid interface by molecular modeling. J. Mol. Struct. (Theochem) 539:279-288.

Creamer, L. K., T. Richardson, and D. A. D. Parry. 1981. Secondary structure of bovine $\alpha_{\mathrm{s} 1^{-}}$and $\beta$-casein in solution. Arch. Biochem. Biophys. 211:689-696.

Eigel, W. N., J. E. Butler, C. A. Ernstrom, H. M. Farrell Jr., V. R. Harwalkar, R. Jenness, and R. McL. Whitney. 1984. Nomenclature of proteins of cow's milk: fifth revision. J. Dairy Sci. 67:1599-1631.

Farrell, H. M., Jr., P. X. Qi, E. M. Brown, P. H., Cooke, M. H. Tunick, E. D. Wickham, and J. J. Unruh. 2002. Molten globule structures in milk proteins: implications for potential new structure-function relationships. J. Dairy Sci. 85:459-471.
Graham, D. E., and M. C. Philips. 1979. Proteins at liquid interfaces. I. Kinetics of adsorption and surface denaturation. J. Colloid Interface Sci. 70:403-414.

Hill, S. E. 1996. Emulsions. Pages 153-185 in Methods of Testing Protein Functionality. G. M. Hall, ed. Blackie, London, U.K.

Husband, F. A., P. J. Wilde, A. R. Mackie, and M. J. Garrood. 1997. A comparison of the functional and interfacial properties of $\beta$ casein and dephosphorylated $\beta$-casein. J. Colloid Interface Sci. 195:77-85.

Jackson, J. D., 1975. Classical Electrodynamics. 2nd ed. Wiley, New York, NY.

de Jongh, H. H. J., and B. de Kruijff. 1990. The conformational changes of apocytochrome c upon binding to phospholipid vesicles and micelles of phospholipid based detergents: a circular dichroism study. Biochim. Biophys. Acta 1029:105-112.

Kabsch, W., and C. Sander. 1983. Dictionary of protein secondary structure: pattern recognition of hydrogen-bonded and geometrical features. Biopolymers 22:2577-2637.

Kumosinski, T. F., E. M. Brown, and H. M. Farrell Jr. 1993. Threedimensional molecular modeling of bovine caseins: an energyminimized $\beta$-casein structure. J. Dairy Sci. 76:931-945.

Mundim, K. C., P. G. Pascutti, M. M. Cassiano, M. Loos, P. M. Bisch. 1998. THOR: a software package for molecular mechanics and dynamics simulations.

Pascutti, P. G., K. C. Mundim, A. S. Ito, and P. M. Bisch. 1999. Polarization effects of peptide conformations at water-membrane interface by molecular dynamics simulation. J. Comput. Chem. 20:971-982.

Press, W. H., S. A. Teukolsky, W. T. Vetterling, and B. P. Flannery. 1992. Numerical Receipes in Fortran. The Art of Scientific Computing. 2nd ed. Cambridge University Press, Cambridge, U.K.

Qi, P. X., E. M. Brown, and H. M. Farrell, Jr. 2001. 'New views' on structure-function relationships in milk proteins. Trends Food Sci. Technol. 12:339-346.

Rankin, S. E., A. Watts, and T. J. T. Pinheiro. 1998. Electrostatic and hydrophobic contributions to the folding mechanism of apocytochrome $\mathrm{c}$ driven by interaction with lipid. Biochemistry 37:12588-12595.

Reitz, J. R., F. J. Milford, and R. W. Christy. 1980. Foundations of Electromagnetism Theory. 3rd ed. Addison-Wesley, Reading, MA.

Ribadeau Dumas, B., G. Brignon, F. Grosclaude, and J.-C. Mercier. 1972. Structure primaire de la caséine $\beta$ bovine. Séquence complète. Eur. J. Biochem. 25:505-514.

Sayle, R. A., and E. J. Milner-White. 1995. RASMOL: biomolecular graphics for all. Trends Biochem. Sci. 20:374-376.

Sood, S. M., and C. W. Slattery. 2000. Association of the quadruply phosphorylated $\beta$-casein from human milk with the nonphosphorylated form. J. Dairy Sci. 83:2766-2770.

Swaisgood, H. E. 1992. Chemistry of the Caseins. Pages 63-110 in Advanced Dairy Chemistry-1: Proteins. P. F. Fox, ed. Elsevier, London, U.K.

Swaisgood, H. E. 1993. Symposium: genetic perspectives on milk proteins: comparative studies and nomenclature. Review and update of casein chemistry. J. Dairy Sci. 76:3054-3061.

SYBYL: molecular modeling package. Tripos, Inc., St. Louis, MO.

van Gunsteren, W. F., and H. J. C. Berendsen. 1987. Groningen molecular simulation (GROMOS) library manual. Biomos, Groningen, The Netherlands.

van Gunsteren, W. F., and H. J. C. Berendsen. 1990. Computer simulation of molecular dynamics: methodology, applications, and perspectives in chemistry. Angew. Chem. Int. Ed. Engl. 29:992-1023.

van Hekken, D. L., and E. D. Strange. 1993. Functional properties of dephosphorylated bovine whole casein. J. Dairy Sci. 76:3384-3391. 